\title{
Role of Protein Kinase $C$ in the Induction and Maintenance of Serotonin-Dependent Enhancement of the Glutamate Response in Isolated Siphon Motor Neurons of Aplysia californica
}

\author{
Greg Villareal, ${ }^{1 \star}$ Quan Li, ${ }^{4 \star}$ Diancai Cai, ${ }^{4}$ Ann E. Fink, ${ }^{2}$ Travis Lim, ${ }^{5}$ Joanna K. Bougie, ${ }^{5}$ Wayne S. Sossin,, 5 and \\ David L. Glanzman ${ }^{1,3,4}$ \\ ${ }^{1}$ Department of Neurobiology, ${ }^{2}$ Interdepartmental Graduate Program in Neuroscience, and ${ }^{3}$ Brain Research Institute, David Geffen School of Medicine at \\ UCLA, University of California, Los Angeles, Los Angeles, California 90095-1761, ${ }^{4}$ Department of Physiological Science, UCLA College of Letters and \\ Science, University of California, Los Angeles, Los Angeles, California 90095-1606, and 5'Department of Neurology and Neurosurgery, Montreal \\ Neurological Institute, McGill University, Montreal, Quebec H3A 2B4, Canada
}

Serotonin (5-HT) mediates learning-related facilitation of sensorimotor synapses in Aplysia californica. Under some circumstances 5-HT-dependent facilitation requires the activity of protein kinase C (PKC). One critical site of PKC's contribution to 5-HT-dependent synaptic facilitation is the presynaptic sensory neuron. Here, we provide evidence that postsynaptic PKC also contributes to synaptic facilitation. We investigated the contribution of PKC to enhancement of the glutamate-evoked potential (Glu-EP) in isolated siphon motor neurons in cell culture. A 10 min application of either 5-HT or phorbol ester, which activates PKC, produced persistent ( $>50 \mathrm{~min})$ enhancement of the Glu-EP. Chelerythrine and bisindolylmaleimide-1 (Bis), two inhibitors of PKC, both blocked the induction of 5-HTdependent enhancement. An inhibitor of calpain, a calcium-dependent protease, also blocked 5-HT's effect. Interestingly, whereas chelerythrine blocked maintenance of the enhancement, Bis did not. Because Bis has greater selectivity for conventional and novel isoforms of PKC than for atypical isoforms, this result implicates an atypical isoform in the maintenance of 5-HT's effect. Although induction of enhancement of the Glu-EP requires protein synthesis (Villareal et al., 2007), we found that maintenance of the enhancement does not. Maintenance of 5-HT-dependent enhancement appears to be mediated by a PKM-type fragment generated by calpaindependent proteolysis of atypical PKC. Together, our results suggest that 5-HT treatment triggers two phases of PKC activity within the motor neuron, an early phase that may involve conventional, novel or atypical isoforms of $\mathrm{PKC}$, and a later phase that selectively involves an atypical isoform.

\section{Introduction}

In mammals the protein kinase $\mathrm{C}$ (PKC) family of enzymes consists of three classes: conventional or classical PKCs that require diacylglycerol (DAG), phosphatidylserine, and $\mathrm{Ca}^{2+}$ for their activation; novel PKCs whose activation requires DAG but not $\mathrm{Ca}^{2+}$; and atypical PKCs that do not depend on either DAG or $\mathrm{Ca}^{2+}$ for their activation (Majewski and Iannazzo, 1998). These classes comprise at least 10 major isoforms. The different iso-

Received Aug. 30, 2008; revised March 9, 2009; accepted March 9, 2009.

This work was supported by National Institutes of Health Grants R37 NS029563 and K02 MH067062 (D.L.G.), and F31 NS04556401 (G.V.), as well as by Canadian Institutes of Health Research Grant 1206 (W.S.S.). W.S.S. is supported by a Chercheurs nationaux Award from the Fonds de la recherche en santé du Québec (FRSQ) and is a Williams Dawson Scholar of McGill University. We thank Drs. Tom 0'Dell and Adam Roberts for their helpful comments on this manuscript and Drs. Yali Zhao and Kelsey Martin for providing the PDBu.

${ }^{*}$ G.V. and Q.L. contributed equally to this work.

Correspondence should be addressed to Dr. David L. Glanzman, Gonda (Goldschmied) Neuroscience and Genetics Research Center, University of California, Los Angeles, 695 Young Drive South, Box 951761, Los Angeles, CA 900951761. E-mail: dglanzman@physci.ucla.edu.

G. Villareal's present address: 4830 Rollingfield Drive, San Antonio, TX 78228.

D0I:10.1523/JNEUROSCI.4149-08.2009

Copyright $\odot 2009$ Society for Neuroscience $\quad$ 0270-6474/09/295100-08\$15.00/0 forms of PKC have been shown to subserve a variety of intracellular signaling pathways. PKCs are also present in invertebrates, where they play important roles in neural signaling (Sossin, 2007). Significant evidence indicates that PKC mediates synaptic and behavioral plasticity in the marine mollusk Aplysia californica. Three isoforms of PKC have been cloned from Aplysia, a classical form (Apl I), a novel form (Apl II) (Kruger et al., 1991), and an atypical form (Apl III) (Bougie et al., 2006). Furthermore, PKC activity has been implicated in learning and memory in Aplysia, including long-term memory (Sossin et al., 1994; Hu et al., 2007) and intermediate-term memory (Sutton and Carew, 2000; Sutton et al., 2004; Zhao et al., 2006).

One site of PKC's contribution to plasticity of the sensorimotor synapse in Aplysia is the presynaptic sensory neuron, as shown in a recent study that used fluorescently tagged Apl I and Apl II to examine PKC translocation in living Aplysia neurons (Zhao et al., 2006). A brief (5 min) application of serotonin (5-HT), an endogenous neurotransmitter that mediates behavioral sensitization in Aplysia (Brunelli et al., 1976; Glanzman et al., 1989; Marinesco and Carew, 2002), caused the translocation of the $\mathrm{Ca}^{2+}$ - 
independent Apl II to the cell membrane of the sensory neuron. [Such translocation is required for activation of the classical and novel PKC isoforms (Sossin, 2007).] A single 5 min pulse of 5-HT treatment by itself produces only short-term facilitation (STF, < $30 \mathrm{~min}$ ). However, when a single pulse of 5-HT is paired with four trains ( $10 \mathrm{~Hz}$ for $2 \mathrm{~s}, 1 \mathrm{~min}$ intertrial interval) of action potentials in the sensory neuron, a form of intermediate-term facilitation (ITF) is produced that lasts for $1-3 \mathrm{~h}$ (Sutton and Carew, 2000). Interestingly, Zhao et al. (2006) found that this activity-dependent ITF depends on translocation of Apl I, but not Apl II, within the sensory neuron (see also Shobe et al., 2009).

Zhao et al. (2006) did not report a major contribution from postsynaptic PKC activity to synaptic plasticity in Aplysia (see Discussion). We have previously shown that activityindependent ITF, produced by a $10 \mathrm{~min}$ application of 5-HT in the absence of neuronal activity, depends on several postsynaptic processes, including elevated postsynaptic $\mathrm{Ca}^{2+}$, postsynaptic exocytosis, postsynaptic protein synthesis, and modulation of the trafficking of postsynaptic AMPA-type glutamate receptors (Li et al., 2005; Villareal et al., 2007). Accordingly, we investigated whether a 10 min treatment with 5-HT activates PKC in Aplysia motor neurons and, if so, whether PKC contributes to 5-HTdependent enhancement of the motor neuron's response to glutamate, an effect that involves modulation of AMPAR trafficking (Chitwood et al., 2001).

\section{Materials and Methods}

Cell cultures. Small siphon (LFS) motor neurons (Frost et al., 1988) were individually dissociated from abdominal ganglia of adult Aplysia californica (80-120 g; Alacrity) and isolated in cell culture as described previously (Chitwood et al., 2001). The dissociated neurons were placed into the center well of plastic culture dishes (50 mm in diameter; No. P50G$0-14-\mathrm{F}$, MatTek Corp.); the center well of the dishes was $10 \mathrm{~mm}$ in diameter and had a glass bottom. The cell cultures were 3-5 d old at the time of the experiments.

Electrophysiology. The electrophysiological methods were similar to those used earlier (Chitwood et al., 2001). Briefly, during the electrophysiological recordings the cell cultures were perfused with 50\% L-15/50\% artificial seawater (ASW) at a rate of $\sim 1 \mathrm{cc} / \mathrm{min}$ using a peristaltic pump (Dynamax Model RP-1; Rainin Instruments). The primary perfusion system consisted of two glass pipettes, one used for the inflow and the other for the outflow. The tips of the inflow and outflow pipettes were placed within the central glass-bottomed well on either side of the motor neuron, with each tip $\sim 3 \mathrm{~mm}$ away from the neuron. The bath volume in the culture dish was maintained at $2 \mathrm{cc}$. To aid in maintaining a stable bath volume a secondary perfusion outflow was used. This outflow consisted of a glass pipette with a broken tip; the tip was positioned outside of the glass-bottomed central well, just slightly touching the surface of the bathing medium in the dish. When the bath volume rose $>2 \mathrm{cc}$, the secondary outflow was engaged, and restored the bath volume to the desired volume. The secondary outflow pipette was coupled to a second peristaltic pump (Dynamax, Rainin Instruments), which was run at a slightly faster rate than the pump used for the primary perfusion system. All experiments were performed at room temperature. The cell body of the motor neuron was impaled with a sharp electrode $(20-30 \mathrm{M} \Omega)$ and held at approximately $-85 \mathrm{mV}$ in current clamp mode $(-0.2$ to $-0.6 \mathrm{nA}$ holding current) throughout the experiment. Glutamate was made as a 2 mu solution in $50 \%$ L- $15-50 \%$ ASW with $0.02 \%$ Fast green added to visualize the drug application. The glutamate solution was pressureejected (Pico-Spritzer II, Parker Hannifin) from a micropipette onto the initial segment of the motor neuron's major neurite. The duration and pressure of the ejection pulse used to deliver the glutamate were adjusted at the start of each experiment to evoke an initial response of 7-20 mV from the motor neuron; thereafter, the ejection pulse was held constant throughout the experiment. The glutamate was washed out immediately after application via the primary perfusion system to prevent desensitization of the response.

All drugs were obtained from Calbiochem unless otherwise indicated. 5-HT (Sigma) was prepared fresh daily as a $2 \mathrm{~mm}$ stock solution in ASW, and diluted to a final concentration of $20 \mu \mathrm{M}$ in normal perfusion medium or DMSO-containing medium (see below) just before an experiment. In experiments involving 5-HT the drug was perfused into the cell culture dish after baseline glutamate stimulation for $10 \mathrm{~min}$, after which it was rapidly washed out with normal perfusion medium. A fresh stock solution of phorbol ester 4b-phorbol-12,13-dibutyrate (PDBu; Sigma), first diluted to $10 \mathrm{~mm}$ in $100 \%$ DMSO, then further diluted to $100 \mu \mathrm{M}$ in $100 \%$ DMSO, was diluted in perfusion medium to a final concentration of $100 \mathrm{~nm}$ plus $0.1 \%$ DMSO immediately before the experiments. The $\mathrm{PDBu}$ was applied to the cell cultures for $10 \mathrm{~min}$. Both chelerythrine and emetine (Sigma) were made into stock solutions (1 mM in deionized water), and then diluted to a final concentration of $1-3 \mu \mathrm{M}$ in perfusion medium. A fresh stock solution of bisindolylmaleimide I (Bis) (100-500 $\mu \mathrm{M}$ in $100 \%$ DMSO) was diluted to a final concentration of $100-500 \mathrm{nM}$ plus $0.1 \%$ DMSO in perfusion medium. In experiments that addressed the potential role of PKC in inducing the enhancement of the glutamate response, chelerythrine was added coincidently with $5-\mathrm{HT}$, whereas Bis was added $10 \mathrm{~min}$ before the 5-HT treatment and then washed out together with the 5-HT. The calpain inhibitor $N$-acetyl-Leu-Leu-Norleu-al (ALLN) (Hiwasa et al., 1990; Khoutorsky and Spira, 2005) was dissolved in deionized water to a final concentration of $100 \mu \mathrm{M}$ and added to the cell cultures coincident with 5-HT. For experiments that addressed the potential role of PKC or protein synthesis in the maintenance of 5-HTdependent enhancement, the PKC inhibitor or protein synthesis inhibitor was applied $15 \mathrm{~min}$ after 5-HT washout; chelerythrine was washed out after $10 \mathrm{~min}$, whereas Bis and emetine were left in the culture dish for the rest of the experiment. For those experiments in which a drug was dissolved in DMSO, the vehicle solution in the 5-HT-alone and control experiments contained the equivalent concentration of DMSO.

PKC purification and assays. PKC Apl III was cloned using degenerate PCR and rapid amplification of cDNA ends (RACE) PCR (Bougie et al., 2009). PKC Apl III and a PKM version of PKC Apl III beginning in the hinge domain with the sequence MKVEQY were cloned into BBACHis2 and baculoviruses were generated as described previously for PKC Apl II (Lim and Sossin, 2006). PKCs were purified on nickel columns as described (Lim and Sossin, 2006); the purity was confirmed by Coomassie staining (data not shown). Purified PKC preparations were diluted until the kinase assay was in the linear range.

Statistical analyses. Peak amplitudes of the evoked glutamate potentials (Glu-EPs) in motor neurons were normalized to the mean amplitude of the 60 Glu-EPs immediately before application of 5-HT, 5-HT/ drug, or vehicle, and were expressed as percentage mean \pm SEM. Parametric tests were used for all statistical analyses. A one-way ANOVA was performed on the data for the $40 \mathrm{~min}$ time point, followed by Tukey post hoc tests for pairwise comparisons (see Villareal et al., 2007). Statistical significance of the group differences was assessed using Graph Pad software. Differences were judged significant at $p<0.05$.

\section{Results PDBu enhances the glutamate response in isolated motor neurons}

Phorbol esters are pharmacological analogs of diacylglycerol (DAG); they mimic DAG by directly binding to the $\mathrm{C} 1$ domains of the PKC holoenzyme, thereby releasing the catalytic domain from suppression (Sossin, 2007). In Aplysia, the phorbol ester $\mathrm{PDBu}$ has been shown to facilitate Aplysia sensorimotor synapses, and this effect is mediated by PKC (Braha et al., 1990; Houeland et al., 2007). In addition, application of PDBu has been shown to translocate fluorescently tagged PKC Apl I and PKC Apl II isoforms from the cytoplasm to the cell membrane of motor neurons (Zhao et al., 2006). Here, we tested whether PDBu produced facilitation of the glutamate response of isolated siphon (LFS) motor neurons in dissociated cell culture. As previously described (Chitwood et al., 2001; Villareal et al., 2007), a 10 min 
application of 5-HT $(20 \mu \mathrm{M})$ produced significant enhancement of the glutamateevoked potential (Glu-EP) (Fig. 1). The mean normalized Glu-EP in the 5-HT group, measured at the $40 \mathrm{~min}$ trial (30 min after 5-HT washout; this trial was used for the group statistical comparisons in this and all subsequent experiments), was $146 \pm 11 \%(n=7)$, whereas the mean normalized Glu-EP for the same trial in the control group was $106 \pm 9 \%(n=6)$. A 10 min application of PDBu (100 nM) also enhanced the Glu-EP. The mean normalized PDBu Glu-EP at 40 min was $154 \pm$ $12 \%(n=7)$. An ANOVA indicated that the differences among the three groups were significant $\left(F_{(2,17)}=5.37, p<0.02\right)$. Post hoc comparisons showed that the 40 min Glu-EP in both the 5-HT and PDBu groups was significantly greater than that of the control group $(p<0.05$ for both comparisons). The difference between the Glu-EP in the 5-HT and PDBu groups was not significant $(p>0.05)$. These results are consistent with the notion that a 10 min exposure to 5 - $\mathrm{HT}$ activates $\mathrm{PKC}$ within the siphon motor neurons.

\section{Inhibitors of PKC and calpain block the induction of 5-HT- dependent enhancement of the Glu-EP}

We next tested whether inhibiting PKC activity disrupted 5-HTdependent enhancement of the glutamate response in motor neurons. We used two general inhibitors of PKC, chelerythrine (Herbert et al., 1990) and bisindolylmaleimide I (Bis) (Toullec et al., 1991). As before, a $10 \mathrm{~min}$ treatment with 5 -HT produced prolonged enhancement of the Glu-EP. The mean normalized Glu-EP on the $40 \mathrm{~min}$ trial in the 5 -HT group was $150 \pm 13 \%$ $(n=6)$ (Fig. 2A). Chelerythrine (1-3 $\mu \mathrm{M})$ blocked the effect of 5 -HT on the Glu-EP. The normalized $40 \mathrm{~min}$ Glu-EP in the 5-HT group was greater than that in the groups that received 5-HT plus chelerythrine (5-HT/Chel; $78 \pm 7 \%, n=6$ ), chelerythrine alone (Chel; $80 \pm 6 \%, n=7$ ), or no drug (control; $84 \pm 11 \%, n=5$ ). An ANOVA indicated that the differences among the groups were significant $\left(F_{(3,20)}=14.01, p<0.0001\right)$. Pairwise comparisons using post hoc tests indicated that the following differences were also significant: 5 -HT versus 5-HT/Chel ( $p<0.001)$, 5-HT versus Chel $(p<0.001)$, and 5-HT versus control $(p<0.01)$. None of the other comparisons were statistically significant.

The effect of Bis on the induction of 5-HT-dependent enhancement was similar to that of chelerythrine (Fig. 2 B). Specifically, in the presence of Bis (100-500 nM) 5-HT's effect on the Glu-EP was disrupted. The mean normalized Glu-EP for the 40 min trial in the 5-HT group was $146 \pm 14 \%(n=5)$, whereas it was only $98 \pm 8 \%(n=6)$ in the group that received 5 -HT together with Bis (5-HT/Bis). An ANOVA indicated that the differences among the group data were significant $\left(F_{(3,18)}=4.76, p<\right.$ 0.02 . Post hoc tests confirmed the statistical significance of the differences between the 5-HT-alone Glu-EP and those in the other groups ( $p<0.05$ for all pairwise comparisons). No other differences were significant.

The results presented in Figs. 1 and 2, $A$ and $B$, represent strong evidence that a 10 min application of 5-HT alone stimulates PKC activity within siphon motor neurons. Given this, an important question is the nature of the isoform that is activated

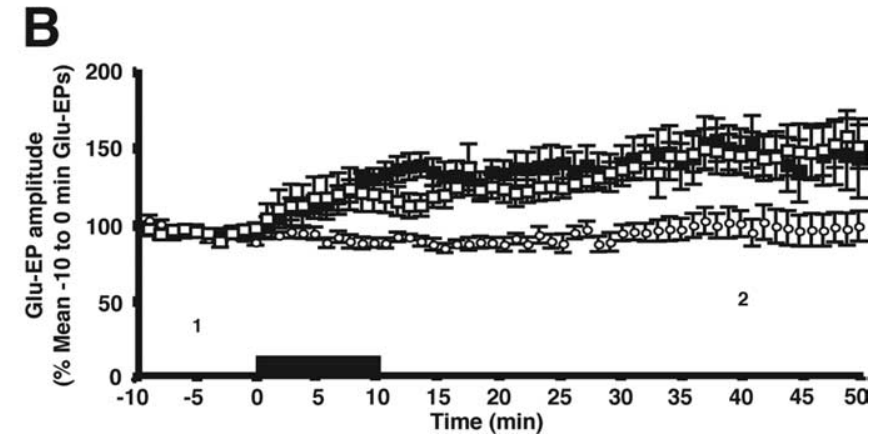

Figure 1. Both 5-HT and phorbol ester enhance the glutamate response in Aplysia motor neurons. $\boldsymbol{A}$, Comparison of the effects of 5-HT and PDBu on the glutamate-evoked potentials (Glu-EPs). Each pair of traces represents responses from a single experia glutamate test pulse once per $10 \mathrm{~s}$; each symbol represents the mean normalized amplitude of six consecutive Glu-EPs. After 10 glutamate stimulation to establish a baseline response, motor neurons received either 5-HT (open squares), PDBu (filled squares), or vehicle alone (control group, open circles). Drug treatment period is indicated by the black bar. The numbers below the data indicate the trials during which the sample Glu-EPs shown in $\boldsymbol{A}$ were recorded. Error bars represent \pm SEM.

by PKC. One likely candidate, because 5-HT elevates intracellular $\mathrm{Ca}^{2+}$ within siphon motor neurons (Chitwood et al., 2001; Li et al., 2005) is the $\mathrm{Ca}^{2+}$-dependent isoform Apl I (Sossin, 2007). However, the 5-HT-stimulated rise of $\mathrm{Ca}^{2+}$ within motor neurons may have other effects as well. One possibility is that 5-HT may stimulate the activity of one or more $\mathrm{Ca}^{2+}$-dependent cysteine proteases (calpains) in motor neurons. This possibility is intriguing, because some isoforms of PKC can be activated through proteolytic cleavage by calpain; the cleavage separates the autoinhibitory regulatory domain from the catalytic domain. The resulting independent catalytic domain, known as PKM, is autonomously active, and can persistently phosphorylate substrates in the absence of second messengers (Sossin, 2007).

To provide initial evidence that an invertebrate PKM contributes to 5-HT's effects on the motor neuron (Bougie et al., 2006, 2007), we tested whether 5-HT-dependent enhancement of the Glu-EP required calpain activity. For this purpose we applied the specific calpain inhibitor ALLN (Hiwasa et al., 1990; Khoutorsky and Spira, 2005) to motor neurons at the same time as the 5-HT. When ALLN $(100 \mu \mathrm{M})$ was applied together with 5-HT the enhancement of the Glu-EP (Fig. 2C) was blocked. The mean normalized Glu-EP following the combined application of 5-HT and ALLN on the $40 \mathrm{~min}$ trial (5-HT/ALLN group) was $107 \pm 6 \%$ $(n=7)$, which was smaller than that after application of 5-HT alone $(168 \pm 25 \%, n=6)$. There was no apparent effect of ALLN alone (ALLN Glu-EP $=102 \pm 1 \%, n=7$; control Glu-EP = $106 \pm 2 \%, n=7)$. An ANOVA indicated that the group differences were significant $\left(F_{(3,23)}=7.26, p<0.002\right)$. Post hoc pairwise comparisons confirmed that the following differences were also significant: 5 -HT versus 5-HT/ALLN $(p<0.01)$, 5-HT versus control $(p<0.01)$, and 5-HT versus ALLN alone $(p<0.01)$.

\section{Chelerythrine disrupts the maintenance of 5-HT-dependent enhancement of the Glu-EP, but Bis does not}

Both chelerythrine and Bis blocked the induction of 5-HT's effect on the glutamate response (Fig. $2 A, B$ ). Therefore, the induction of the enhancement depends on PKC activity. We next asked whether the maintenance of the enhancement requires ongoing PKC activity. Accordingly, we first treated the motor neuron with 5-HT for $10 \mathrm{~min}$ and then, beginning $15 \mathrm{~min}$ after washout of the 


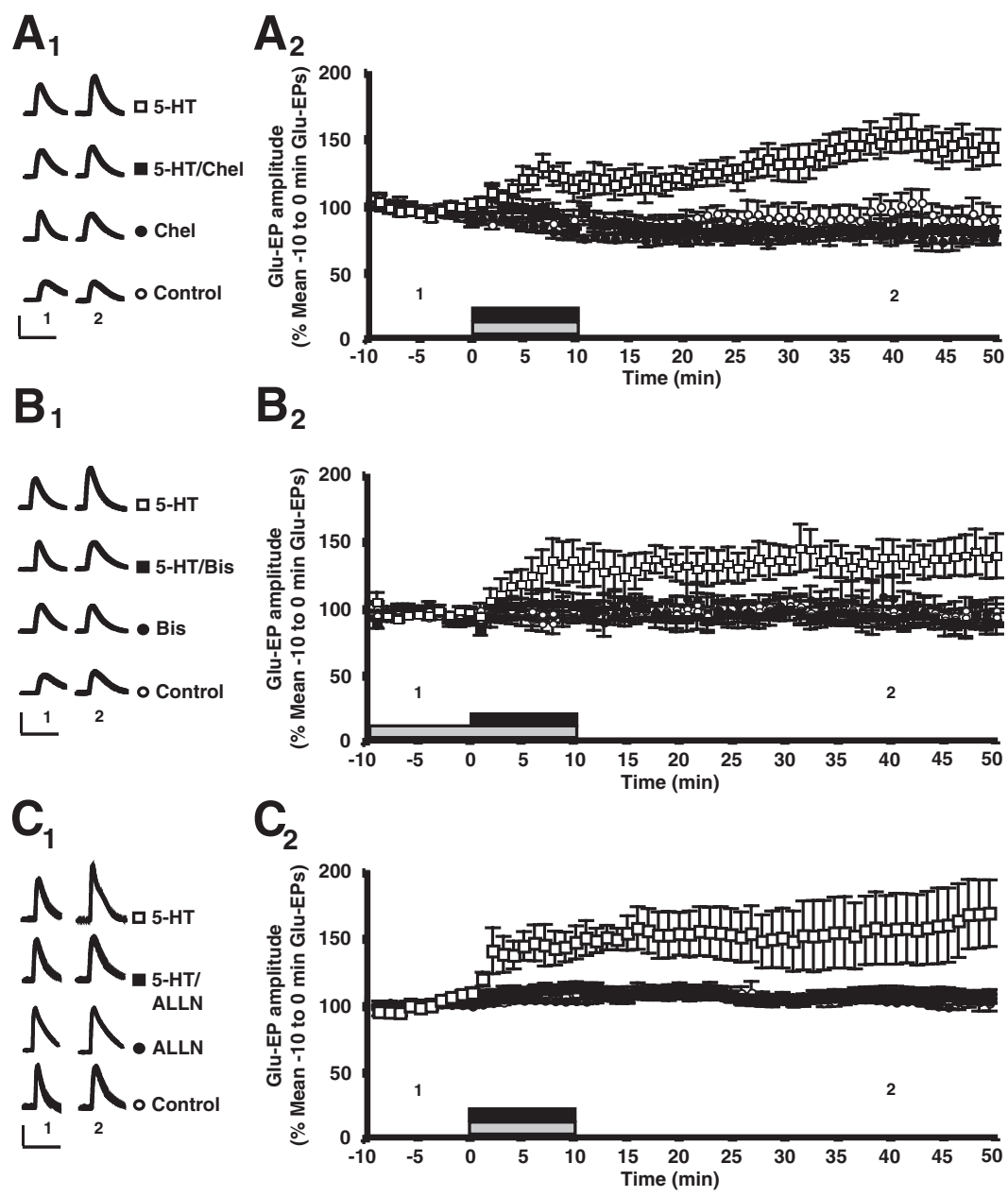

Figure 2. Inhibition of either PKC or calpain blocks the induction of 5-HT-dependent enhancement of the glutamate response. $A_{1}$, Effect of the PKC inhibitor chelerythrine on the induction of enhancement of the Glu-EP. The figure presents sample traces from a single experiment for each experimental group. Note that in the control experiments, no drug was applied. Traces marked " 1 " represent Glu-EPs evoked 5 min before the onset of the 10 min period of drug treatment (or 5 min after the start of testing); those marked " 2 " represent Glu-EPs evoked $30 \mathrm{~min}$ after drug washout (or $50 \mathrm{~min}$ after the start of testing). Calibration: $A_{1}, \boldsymbol{B}_{1}, \boldsymbol{C}_{1}, 10$ $\mathrm{mV}, 500 \mathrm{~ms} . A_{2}$, Summary of the experiments showing the effect of chelerythrine on induction. Motor neurons received either 5-HT alone (open squares), 5-HT combined with chelerythrine (filled squares), chelerythrine alone (Chel; filled circles), or perfusion medium alone (controls; open circles). The black bar indicates the period of 5 - HT treatment; the gray bar indicates the period of chelerythrine treatment. The numbers below the data indicate the trials during which the sample Glu-EPs shown in $A_{1}$ were recorded. Error bars represent \pm SEM. $\boldsymbol{B}_{1}$, Effect of another PKC inhibitor, bisindolylmaleimide-1 (Bis), on the induction of enhancement of the glutamate response. Shown are sample traces from a single experiment for each experimental group. In the control experiments only the vehicle was applied. The Bis was applied for $20 \mathrm{~min}$, and was present for $10 \mathrm{~min}$ before the onset of the 10 min period of 5 -HT or treatment, which occurred 10 min after the start of testing. Traces marked " 1 " represent Glu-EPs evoked $5 \mathrm{~min}$ before the onset of 5 -HT or vehicle (or $5 \mathrm{~min}$ after the start of testing); those marked "2" represent Glu-EPs evoked 30 min after washout of the drug or vehicle (or 50 min after the start of testing). $\boldsymbol{B}_{2}$, Summary of the experiments showing block of the induction of 5-HT-dependent enhancement by Bis. Motor neurons were treated with 5-HT (open squares), 5 -HT and Bis (filled squares), Bis alone (filled circles), or vehicle alone (control; open circles). The black bar indicates the 5 -HT treatment period, whereas the gray bar indicates the Bis treatment period. The numbers below the data indicate the trials at which the sample Glu-EPs shown in $\boldsymbol{B}_{1}$ were recorded. Error bars represent \pm SEM. $C_{1}$, Effect of the calpain inhibitor ALLN on the induction of enhancement. The figure shows sample traces from a single experiment for each experimental group. In the control experiments no drug was applied. Traces marked " 1 " represent Glu-EPs evoked 5 min before the onset of drug treatment (or 5 min after the start of testing); those marked "2" represent Glu-EPs evoked 30 min after drug washout (or 50 min after the start of testing). $\boldsymbol{C}_{2}$, Summary of the experiments showing the block of enhancement of the Glu-EP by inhibition of calpain activity. Motor neurons received 5-HT (open squares), 5-HT plus ALLN (filled squares), ALLN alone (filled circles), or perfusion medium alone (control; open circles). The black bar indicates the 5-HT treatment period, whereas the gray bar indicates the ALLN treatment period. The numbers below the data indicate the trials at which the sample Glu-EPs shown in $\boldsymbol{C}_{\text {}}$ were recorded. Error bars represent \pm SEM.

5-HT, applied either chelerythrine or Bis to the culture dish. (The chelerythrine was left in the dish for only $10 \mathrm{~min}$, whereas the Bis was left in for $25 \mathrm{~min}$.) Chelerythrine eliminated the 5-HTinduced enhancement of the Glu-EP within 5 min after introduc- tion of the drug (Fig. 3A). The mean normalized EPSP for the $40 \mathrm{~min}$ trial was $145 \pm 9 \%(n=6)$ in the 5 -HT group, whereas it was $96 \pm 17 \%(n=4)$ in the group in which chelerythrine was applied after 5-HT (5-HT/Chel group). The application of chelerythrine alone had no apparent effect on the Glu-EP (Chel Glu$\mathrm{EP}=92 \pm 4 \%, n=6$ ), as indicated by the similarity of the results in Chel group with those in the control group (Glu-EP $=86 \pm$ $10 \%, n=4)$. Note that the amplitude of the Glu-EP did not recover after washout of chelerythrine in the 5-HT/Chel experiments. These group differences were significant, as indicated by an ANOVA $\left(F_{(3,16)}=8.69, p<0.002\right)$. Pairwise comparisons using post hoc tests indicated that the Glu-EP in the 5-HT group was significantly more enhanced at 40 min than those in the other three groups ( $p<0.05$ for each comparison).

Unlike chelerythrine, Bis did not affect the maintenance of the 5-HT-induced enhancement of the Glu-EP (Fig. 3B). There was no difference between the $40 \mathrm{~min}$ Glu-EP in the 5 -HT group (146 $\pm 14 \%$, $n=5)$ and that in the group treated with Bis after 5-HT (5-HT/Bis Glu-EP = $131 \pm$ $10 \%, n=6)$. The glutamate response was enhanced in both groups compared with that in the Bis-alone (Bis Glu-EP $=99 \pm$ $6 \%, n=6$ ) and control (Glu-EP $=100 \pm$ $10 \%, n=6)$ groups. These findings were confirmed by the results of the statistical analyses. The results from the ANOVA indicated that the group differences were significant $\left(F_{(3,19)}=5.32, p<0.01\right)$. The post hoc tests confirmed the lack of a significant difference between the 5-HT and 5-HT/ Bis groups ( $p>0.05)$, as well as the significance of the differences between the 5-HT group and the Bis and control groups ( $p<$ 0.05 in each case).

It has been previously reported that chelerythrine selectively blocks a mammalian form of PKM, PKM $\zeta$, at low concentrations (Ling et al., 2002). We therefore investigated the effect of both chelerythrine and Bis on the recently sequenced Aplysia PKM isoform, PKM Apl III (Bougie et al., 2006, 2007). PKM Apl III is formed by calpain-dependent cleavage from PKC III (Bougie et al., 2009). We compared the inhibitory effects of chelerythrine and Bis on PKC Apl II, the novel form of PKC in Aplysia (Sossin et al., 1993), as well as on PKC Apl III and PKM Apl III. [We did not test the effects of the two inhibitors on PKC Apl I. It has been previously shown that the inhibitors do not distinguish between Apl I and Apl II (Sossin, 1997; Manseau et al., 2001).] His-tagged Aplysia PKC Apl II, PKC Apl III and a PKM form of PKC Apl III were expressed in SF9 cells 
using baculovirus and purified on nickel columns (Lim and Sossin, 2006). Kinase assays were as described with $50 \mu \mathrm{M}$ adenosine triphosphate (ATP) (Lim and Sossin, 2006), but $10 \mu \mathrm{M} \varepsilon$ substrate $\left(\mathrm{H}_{2} \mathrm{NOERMRPRLRQGSVRRRV-OH)} \mathrm{was}\right.$ used because this substrate is equally well phosphorylated by novel and atypical PKCs (Ling et al., 2002) (data not shown). The kinase assays used the activators phosphatidylserine (PS) and the phorbol ester, 12-O-tetradecanoylphorbol-13-acetate (TPA). These are required for PKC Apl II activity (Sossin et al., 1996). PKM Apl III activity was not affected by PS, whereas PS caused twofold stimulation of PKC Apl III activity. (Presumably, this difference is due to the $\mathrm{C} 1$ domain that is present in PKC Apl III, but absent in PKM Apl III.) TPA had no effect on either PKM Apl III or PKC Apl III.

We observed that chelerythrine equally inhibited PKC Apl II and PKC Apl III, but inhibited the PKM Apl III at much lower concentrations (Fig. $3 C_{1}$ ), within the range of those used in the electrophysiological experiments $(1-5 \mu \mathrm{M})$. Bis inhibited PKC Apl II at low concentrations, had no significant effect on PKC Apl III even at high concentrations (up to $50 \mu \mathrm{M}$ ), and inhibited PKM Apl III, but only at concentrations $(5-10 \mu \mathrm{M})$ significantly higher than those used for electrophysiology (100$500 \mathrm{~nm}$ ) (Fig. $3 C_{2}$ ). The greater inhibitory effect of chelerythrine and Bis on PKM Apl III than on PKC Apl III is probably due to the loss of the regulatory subunit in PKM Apl III, which may serve to block access of the inhibitors to their binding sites in PKC Apl III (Sossin, 2007). In summary, we found that PKC Apl III activity was not blocked by Bis, but was blocked by chelerythrine. Moreover, the PKM version of PKC Apl III was particularly sensitive to low concentrations of chelerythrine, similar to those reported as effective in inhibiting mammalian PKM $\zeta$ (Ling et al., 2002).

\section{Maintenance of 5-HT-dependent} enhancement does not require ongoing protein synthesis

We have recently reported that the induction of 5-HT's effect on the Glu-EP depends on rapid, local protein synthesis (Villareal et al., 2007) (Fig. 4A). Accordingly, we wished to know whether the maintenance of the 5-HT-dependent enhancement of the glutamate response depends on ongoing protein synthesis. To test this possibility the protein synthesis inhibitor emetine (1-3 $\mu \mathrm{M})$ was applied to the cell cultures starting at $15 \mathrm{~min}$ after washout of 5-HT (immediately before the 25 min trial) (Fig. 4B). Inhibition of protein synthesis had no apparent effect on the concentration.
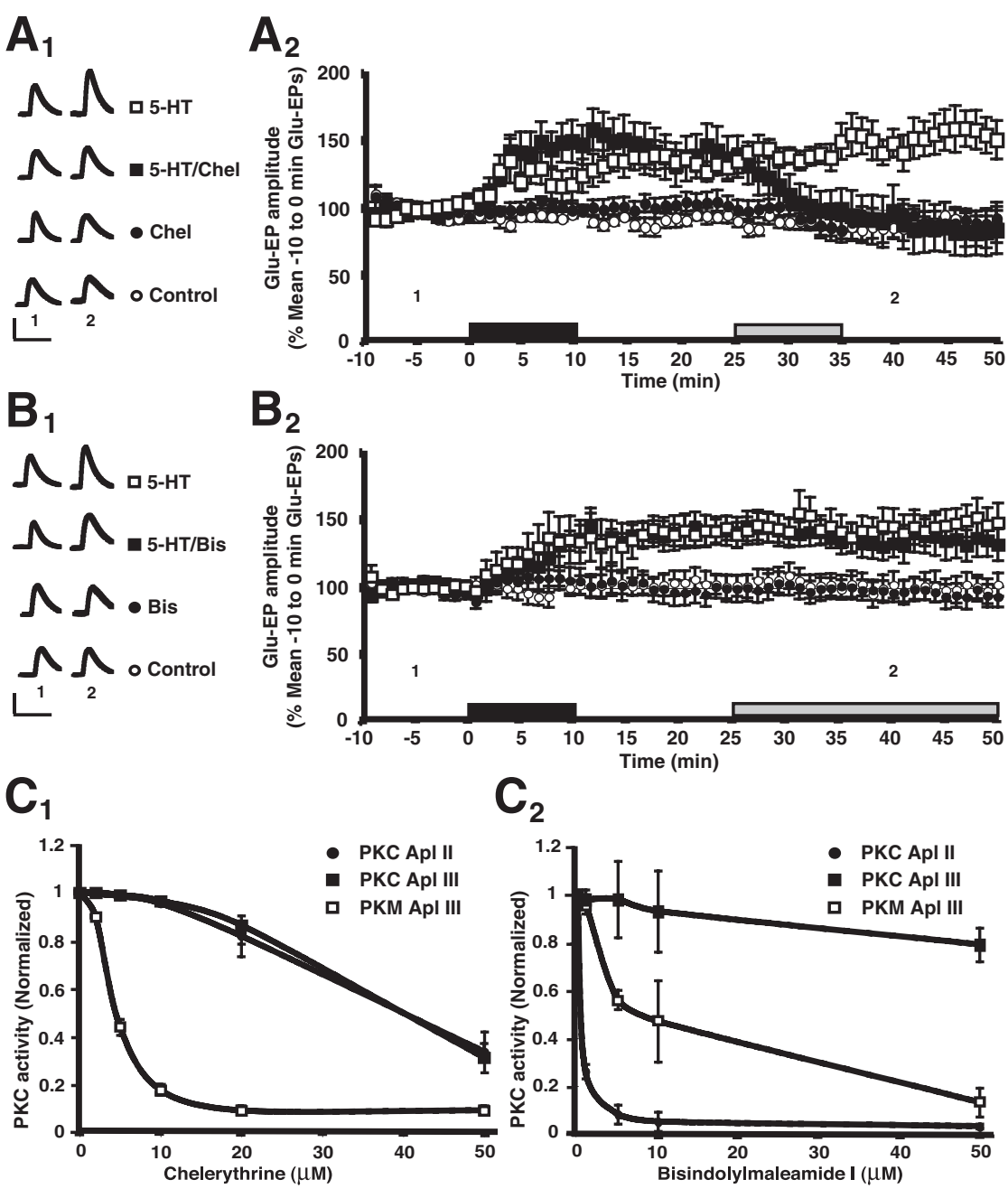

Figure 3. PKM ApI III mediates maintenance of 5-HT-dependent enhancement. $A_{1}$, Effect of chelerythrine on the maintenance of the enhancement. The figure presents sample traces from a single experiment for each experimental group. No drug was applied in the control experiments. Traces marked " 1 " represent Glu-EPs evoked 5 min before the onset of 5-HT treatment (or 5 min after the start of testing); those marked "2" represent Glu-EPs evoked 30 min after washout of 5-HT (or 50 min after the start of testing). In the experiments involving chelerythrine, the inhibitor was applied for $10 \mathrm{~min}$ starting $15 \mathrm{~min}$ after washout of 5 -HT, or during the equivalent time period in the chelerythrine-alone experiments. Calibration: $A_{1}, B_{1}, 10 \mathrm{mV}, 500 \mathrm{~ms}$. $A_{2}$, Summary of the experiments showing that chelerythrine disrupts the maintenance of enhancement. Motor neurons were stimulated with 5-HT alone (open squares), 5-HT followed by chelerythrine (25-35 min trials; filled squares), chelerythrine alone (filled circles), or perfusion medium alone (control; open circles). The black bar indicates the period of 5-HT treatment, whereas the gray bar indicates the chelerythrine treatment period. Notice that the enhancement did not reappear after washout of chelerythrine in the 5-HT/Chel experiments. The numbers below the data indicate the trials at which the sample Glu-EPs shown in $A_{1}$ were recorded. Error bars represent \pm SEM. $\boldsymbol{B}_{1}$, Effect of Bis on the maintenance of 5-HT-dependent enhancement of the Glu-EP. The figure presents sample traces from a single experiment for each experimental group. Traces marked " 1 " represent Glu-EPs evoked 5 min before the onset of 5-HT treatment (or 5 min after the start of testing); those marked "2" represent Glu-EPs evoked 30 min after washout of 5 -HT (or 50 min after the start of testing). In the experiments involving Bis, the inhibitor was applied for 25 min starting 15 min after washout of 5 -HT, or during the equivalent time period in the Bis-alone experiments. In the control experiments the vehicle was applied during the same time that Bis was applied in the 5 -HT/Bis and Bis-alone experiments. $\boldsymbol{B}_{2}$, Summary of the experiments showing the lack of an effect of Bis on maintenance of the enhancement. Cultures were exposed to $5-\mathrm{HT}$ (open squares), 5-HT followed by Bis (25-50 min trials; filled squares), Bis alone (filled circles), or vehicle alone (control; open circles). The black bar indicates the 5-HT treatment period, whereas the gray bar indicates the Bis treatment period. The numbers below the data indicate the trials at which the sample Glu-EPs shown in $\boldsymbol{B}_{\boldsymbol{1}}$ were recorded. Error bars represent \pm SEM. $\boldsymbol{C}_{\boldsymbol{1}}$, Inhibition of PKC ApI II (filled circles), PKC ApI III (filled squares), and PKM ApI III (open squares) by chelerythrine. $\boldsymbol{C}_{2}$, Inhibition of PKC ApI II (filled circles), PKC Apl III (filled squares), and PKM Apl III (open squares) by Bis. Error bars are \pm SEM; $n>3$ for each

ongoing enhancement of the Glu-EP produced by 5-HT. The mean normalized Glu-EP on the 40 min trial after 5-HT treatment alone was $149 \pm 8 \%(n=6)$, whereas the mean normalized Glu-EP in the group treated with 5-HT and then emetine was 


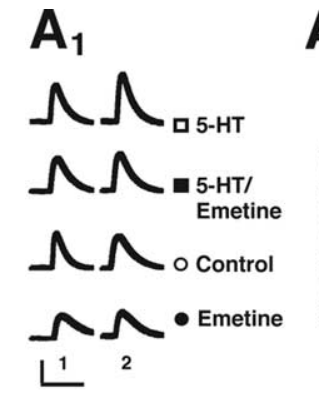

$\mathrm{A}_{2}$

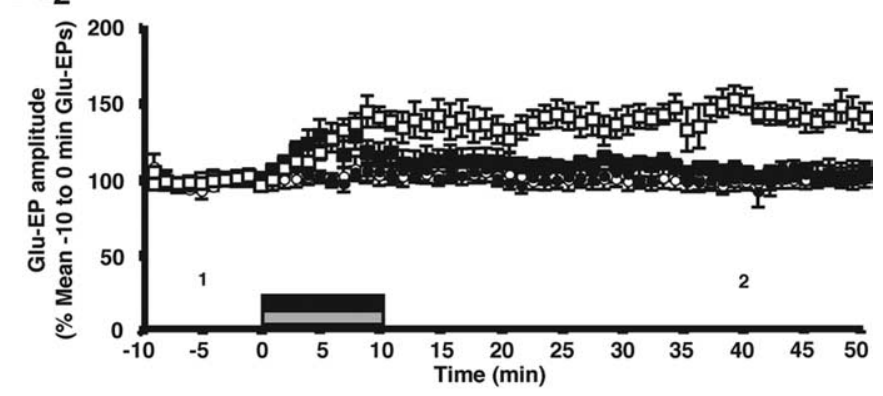

$B_{1}$

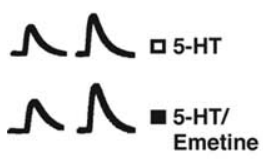

$\Lambda_{L^{1}}{ }_{2} \circ$ Control

$\mathrm{B}_{2}$

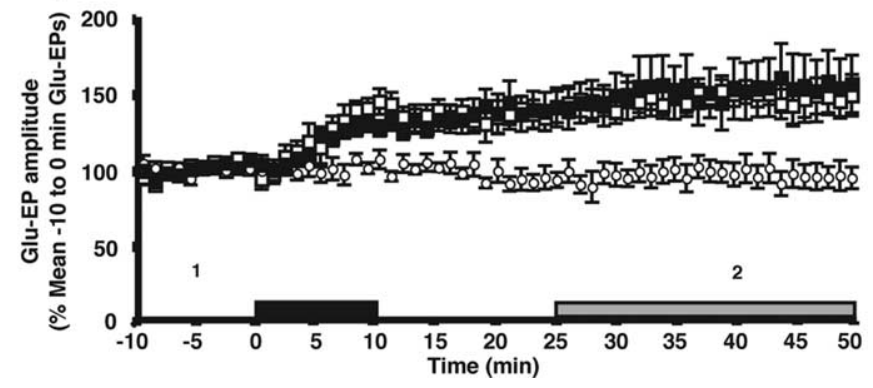

Figure 4. Although induction of the enhancement of the glutamate response requires protein synthesis, maintenance of the enhancement does not depend on ongoing protein synthesis. $\boldsymbol{A}_{1}$, Effect of emetine treatment on the induction of 5 -HT-dependent enhancement of the Glu-EP. The figure presents sample traces from a single experiment for each experimental group. No drug was applied in the control experiments. Traces marked "1" represent Glu-EPs evoked 5 min before the onset of drug treatment (or 5 min after the start of testing); those marked "2" represent Glu-EPs evoked $30 \mathrm{~min}$ after drug washout (or 50 min after the start of testing). Calibration: $\boldsymbol{A}_{1}, \boldsymbol{B}_{1}, 10 \mathrm{mV}, 500 \mathrm{~ms}$. $\boldsymbol{A}_{2}$, Summary of experiments showing that inhibition of protein synthesis blocks the induction of the enhancement of the Glu-EP. Motor neurons received either 5-HT alone (open squares), 5 -HT and emetine (filled squares), emetine alone (filled circles), or perfusion medium alone (control; open circles). The overall mean Glu-EP in the 5-HTalone group $(131 \pm 1 \%, n=8)$ was significantly greater than those in groups treated with 5 -HT and emetine $(109 \pm 1 \%, n=$ 7) and emetine alone $(102 \pm 1 \%, n=6)$, as well as in the control group (101 $\pm 1 \%, n=6)(p<0.05$ for each comparison). The black bar indicates the $5-\mathrm{HT}$ treatment, and the gray bar the emetine treatment. The numbers below the data indicate the trials at which the sample Glu-EPs shown in $\boldsymbol{A}_{1}$ were recorded. Error bars represent \pm SEM. From Villareal et al. (2007). See that study for additional details. $\boldsymbol{B}_{1}$, Effect of emetine treatment on the maintenance of 5-HT-dependent enhancement of the Glu-EP. The figure presents sample traces from a single experiment for each experimental group. No drug was applied in the control experiments. Traces marked "1" represent Glu-EPs evoked 5 min before the onset of 5-HT treatment (or 5 min after the start of testing); those marked " 2 " represent Glu-EPs evoked $30 \mathrm{~min}$ after washout of 5-HT (or $50 \mathrm{~min}$ after the start of testing). $\boldsymbol{B}_{\mathbf{2}}$, Summary of experiments showing that protein synthesis is not required for the maintenance of 5 -HT-dependent enhancement. Motor neurons received either 5-HT alone (open squares), 5-HT followed by emetine (filled squares), or perfusion medium alone (control, open circles). In the experiments involving emetine, the inhibitor was applied for 25 min starting 15 min after washout of 5-HT. The black bar indicates the 5-HT treatment period, and the gray bar indicates the emetine treatment period. The numbers below the data indicate the trials at which the sample Glu-EPs shown in $\boldsymbol{B}_{1}$ were recorded. Error bars represent $\pm \mathrm{SEM}$.

$144 \pm 20 \%(n=5)$. The mean normalized Glu-EP in the control group was $99 \pm 7 \%(n=7)$. An ANOVA confirmed that the group differences were significant $\left(F_{(2,17)}=5.37, p<0.02\right)$. Post hoc tests indicated that the mean Glu-EPs in both the 5-HT and 5-HT/ Emetine groups were larger than that for the control group ( $p<0.05$ for each comparison), and that the difference between the Glu-EPs in the 5-HT and 5-HT/Emetine groups was not significant $(p>0.05)$.

\section{Discussion}

We have shown that PKC plays a critical role in 5-HT-dependent enhancement of the glutamate response in Aplysia motor neurons. Previous work has shown that this form of plasticity depends on release of $\mathrm{Ca}^{2+}$ from intracellular stores, exocytosis, and rapid, local protein synthesis, and results in the functional upregulation of AMPA-type receptors in the motor neuron (Chitwood et al., 2001; Villareal et al., 2007). Furthermore, 5-HTelicited processes in the motor neuron contribute significantly to activity-independent ITF of the sensorimotor synapse, as well as to behavioral dishabituation and sensitization of the siphon withdrawal reflex (Li et al., 2005). Together with the prior results, the present results suggest that postsynaptic PKC mediates activity-independent ITF and behavioral enhancement in Aplysia.

The contribution of PKC to 5-HTdependent plasticity in the present experiments appears to involve at least two distinct isoforms: one and possibly more that mediate the induction of plasticity, and an atypical PKM that mediates maintenance. The evidence for a non-atypical PKC in induction includes the ability of $\mathrm{PDBu}-$ which activates PKC Apl I and PKC Apl II but does not affect the atypical PKC Apl III-to increase the Glu-EP, and the ability of Bis - which inhibits PKC Apl I and PKC Apl II but does not inhibit the atypical $\mathrm{PKC}$ - to block induction. In contrast, chelerythrine is a much more effective inhibitor of PKM Apl III than of PKC Apl I or PKC Apl II (Fig. 3C $_{1}$ ) (Ling et al., 2002; Sossin, 2007). Because PKC Apl I depends on $\mathrm{Ca}^{2+}$ for its activation (Sossin et al., 1993, 2007), and because a $10 \mathrm{~min}$ exposure to 5-HT elevates intracellular $\mathrm{Ca}^{2+}$ within the motor neuron through release from intracellular stores (Chitwood et al., 2001; Li et al., 2005), we favor the idea that the induction of 5-HT-dependent enhancement of the glutamate response is mediated, at least in part, by PKC Apl I. In support of this idea, Zhao et al. (2006) reported that application of $\mathrm{PDBu}$, which we found to mimic the effect of 5-HT on the Glu-EP, produced significant translocation of green fluorescent protein (GFP)-tagged Apl I from the cytoplasm to the plasma membrane in LFS motor neurons.

The block of induction by calpain was rapid and, importantly, we observed no early calpain-independent phase of enhancement (Fig. 2C). The simplest explanation for our results is that PKC Apl I and calpain act together to stimulate proteolysis of PKM Apl III, and that production of this isoform is sufficient to increase the Glu-EP. [PKM $\zeta$ is also sufficient to increase AMPA receptor-mediated synaptic currents in area CA1 of the hippocampus (Ling et al., 2002; Yao et al., 2008).] However, we cannot rule out the possibility that actions of PKC Apl I or calpain that are independent of PKM Apl III formation support the early phase of the 5-HT-mediated increase in the Glu-EP.

The requirement for activation of PKC Apl I within the motor neuron during 5-HT-dependent enhancement of the Glu-EP conflicts with the results of Zhao and colleagues (2006), who found that a 5 min treatment of 5-HT did not produce translocation of either GFP-tagged Apl I or Apl II in LFS motor neurons. Possibly, due to lack of sensitivity of their fluorescence methodology, Zhao et al. missed the 5-HT-stimulated translocation of Apl I or Apl II in motor neurons in their study (despite detecting 
PDBu-stimulated translocation); for example, their methodology may not have been sufficiently sensitive to detect changes in the activity of PKC isoforms in signaling microdomains in the motor neuron plasma membrane (Mager et al., 2007). The idea that 5-HT stimulates translocation of PKC within Aplysia motor neurons is supported by data from Fulton et al. (2008), who found that 5-HT-dependent enhancement of the Glu-EP in isolated motor neurons requires phospholipase C (PLC) activity. PLC activity, through DAG production, activates both classical and novel PKC isoforms (Sossin, 2007). Furthermore, Zhao et al. (2006) did not test the effect of a 10 min 5-HT exposure on postsynaptic PKC activity. A 5 min application of 5-HT induces only short-term facilitation (STF; lasting $<30 \mathrm{~min}$ ) of the sensorimotor synapse; in contrast, a $10 \mathrm{~min}$ application of 5-HT induces activity-independent ITF (lasting $>30 \mathrm{~min}$ and $<3 \mathrm{~h})(\mathrm{Li}$ et al., 2005) (see Sutton and Carew, 2000). A fundamental mechanistic distinction between STF and ITF may be that ITF recruits postsynaptic PKC activity whereas STF does not. We have previously suggested (Li et al., 2005) that STF relies on exclusively presynaptic processes, whereas ITF relies on both presynaptic and postsynaptic processes. If so, one might expect there to be an early stage of facilitation of the sensorimotor synapse that is independent of calpain-stimulated postsynaptic PKM Apl III activity. [Notice that $\mathrm{PKM} \zeta$ activity does not contribute to the early phase of LTP in the CA1 region of the hippocampus, but is required for intermediate and late phases of LTP (Serrano et al., 2005).]

In contrast to induction, maintenance of 5-HT-dependent enhancement appears to require PKM Apl III activity, because the maintenance is blocked by chelerythrine (Fig. $3 A$ ). Importantly, our bioassay measurements indicated that chelerythrine was an effective inhibitor of PKM at the low concentrations (1-5 $\mu \mathrm{M})$ used in the electrophysiological experiments, whereas Bis was ineffective at inhibiting PKM at concentrations (100-500 $\mathrm{nM}$ ) used for electrophysiology (Fig. 3C). Recent evidence indicates that in Aplysia the PKM fragment is generated by calpaindependent cleavage from the atypical PKC (Bougie et al., 2009). In support of this idea, we observed that an inhibitor of calpain, ALLN, blocked the induction of enhancement of the glutamate response when applied together with 5-HT (Fig. 2C).

Sutton et al. (2004) have reported that persistent activity of a PKM-like isoform of PKC mediates site-specific intermediateterm sensitization memory (ITM) in Aplysia. This type of behavioral memory was demonstrated in a reduced preparation by testing a specific site on the preparation's tail after delivering a shock to the test site. Activity-dependent ITM appears to differ, mechanistically, from activity-independent ITF (Li et al., 2005). First, the induction of activity-dependent ITM does not depend on protein synthesis (Sutton et al., 2004), in contrast to activityindependent ITF (Villareal et al., 2007). Second, although the maintenance of activity-dependent ITM is disrupted by chelerythrine, the memory returns as soon as the inhibitor is washed out [Sutton et al. (2004), their Fig. 4A]. This finding contrasts with our observation that 5-HT-induced enhancement of the Glu-EP did not reappear after washout of chelerythrine (Fig. $3 A$ ). A potential explanation for the differing results in the two studies is that the behavioral change is regulated predominantly by another PKM-type isoform of PKC, one whose activity is not permanently disrupted by $15 \mathrm{~min}$ of chelerythrine/Bis treatment.

Recent work in the mammalian hippocampus indicates that the persistent form of LTP, referred to as late-phase LTP (L-LTP) is maintained by $\mathrm{PKM} \zeta$, an isoform derived from the gene for an atypical mammalian PKC, PKC $\zeta$ (Ling et al., 2002; Hernandez et al., 2003; Pastalkova et al., 2006). Activity-independent ITF and
L-LTP have a maintenance mechanism in common, specifically, upregulation of postsynaptic AMPA receptor function (Ling et al., 2002; Li et al., 2005; Yao et al., 2008). In the hippocampus $\mathrm{PKM} \zeta$ appears to direct postsynaptic AMPA receptor trafficking (Yao et al., 2008), and this is a likely role for PKM Apl III in Aplysia as well (Chitwood et al., 2001; Glanzman, 2007, 2008). One apparent difference between mammalian PKM $\zeta$ and the PKM version of PKC Apl III in Aplysia is that PKM $\zeta$ is not formed by proteolysis; rather, $\mathrm{PKM} \zeta$ is generated by local translation from PKM $\zeta$ mRNA (Hernandez et al., 2003). However, the alternative transcriptional start site required for formation of the $\mathrm{PKM} \zeta$-specific mRNA and the initiating methionine for PKM $\zeta$ are only found in chordates, and probably emerged after the duplication of an ancestral atypical PKC into separate $\zeta$ and $\iota$ isoforms (Bougie et al., 2009). Therefore, although the regulation of plasticity by an atypical PKM may be highly conserved, it is possible that the mechanism for formation of this kinase has changed over evolution. On the other hand, calpain is also required for some forms of learning-related synaptic plasticity in the mammalian brain (Lu et al., 2001); consequently, cleavage-formed PKM may contribute to synaptic plasticity in mammals as well.

Despite potential differences in the formation and regulation of the mammalian and molluscan PKM fragments, their roles in synaptic plasticity and learning appear remarkably similar. In particular, disruption of the ongoing activity of $\mathrm{PKM} \zeta$ by $\zeta$ inhibitory peptide (ZIP) appears to "erase" both established synaptic plasticity and learning (Ling et al., 2002; Pastalkova et al., 2006; Shema et al., 2007) in mammals. Similarly, in the present experiments we found evidence for erasure of persistent plasticity in Aplysia motor neurons through inhibition of PKM Apl III by chelerythrine (Fig. 3A). The precise mechanisms that underlie the erasure of memory/synaptic plasticity in mammals are unclear at present. One possibility is that the ongoing activity of $\mathrm{PKM} \zeta$ is critical for maintaining additional AMPA receptors in postsynaptic membranes (Ling et al., 2006; Yao et al., 2008); inhibiting $\mathrm{PKM} \zeta$ may cause recently inserted AMPA receptors to be endocytosed and unavailable for trafficking when the enzyme is no longer inhibited after washout of ZIP/chelerythrine. Another possibility is that $\mathrm{PKM} \zeta$ is prone to proteolysis in the inhibited state. Similar phenomena may explain the apparent irreversibility of the disruption of enhancement of the Glu-EP following washout of chelerythrine in our study. It will be important to determine in future experiments whether persistent synaptic plasticity in Aplysia, particularly long-term facilitation (LTF) (Montarolo et al., 1986), can be permanently erased by inhibition of PKM Apl III. In this regard, it has been found that, although chelerythrine did not block the induction of $24 \mathrm{~h} \mathrm{LTF}$ by five spaced pulses of 5HT (Hu et al., 2003, 2004), a 2 h application of chelerythrine, applied after the five pulses of 5-HT, did block the $24 \mathrm{~h} \mathrm{LTF}$ (Hu et al., 2007). These are intriguing results, because they implicate PKM Apl III in the maintenance of at least one form of LTF. The Aplysia sensorimotor synapse in cell culture may offer an advantageous preparation for a rigorous analysis of the mechanisms underlying erasure of longterm synaptic plasticity through inhibition of PKM.

\section{References}

Bougie J, Lim T, Ferraro G, Manjunath V, Scott D, Sossin WS (2006) Cloning and characterization of protein kinase C (PKC) Apl III, a homologue of atypical PKCs in Aplysia. Soc Neurosci Abstr 32:669.10.

Bougie JK, Lim T, Manjunath V, Farah-Abi C, Nagakura I, Sossin WS (2007) The role of atypical protein kinase $\mathrm{C}$ (PKC) zeta in synaptic plasticity in Aplysia. Soc Neurosci Abstr 33:208.5.

Bougie JK, Lim T, Farah CA, Manjunath V, Nagakura I, Ferraro GB, Sossin WS (2009) The atypical protein kinase C in Aplysia can form a PKM by 
cleavage. J Neurochem. Advance online publication. Retreived April 10, 2009. doi:10.1111/j.1471-4159.2009.06045.x.

Braha O, Dale N, Hochner B, Klein M, Abrams TW, Kandel ER (1990) Second messengers involved in the two processes of presynaptic facilitation that contribute to sensitization and dishabituation in Aplysia sensory neurons. Proc Natl Acad Sci U S A 87:2040-2044.

Brunelli M, Castellucci V, Kandel ER (1976) Synaptic facilitation and behavioral sensitization in Aplysia: possible role of serotonin and cyclic AMP. Science 194:1178-1181.

Chitwood RA, Li Q, Glanzman DL (2001) Serotonin facilitates AMPA-type responses in isolated siphon motor neurons of Aplysia in culture. J Physiol 534:501-510.

Frost WN, Clark GA, Kandel ER (1988) Parallel processing of short-term memory for sensitization in Aplysia. J Neurobiol 19:297-334.

Fulton D, Condro MC, Pearce K, Glanzman DL (2008) The potential role of postsynaptic phospholipase $\mathrm{C}$ activity in synaptic facilitation and behavioral sensitization in Aplysia. J Neurophysiol 100:108-116.

Glanzman DL (2007) Simple minds: the neurobiology of invertebrate learning and memory. In: Invertebrate neurobiology (North G, Greenspan RJ, eds), pp 347-380. New York: Cold Spring Harbor Laboratory.

Glanzman DL (2008) New tricks for an old slug: the critical role of postsynaptic mechanisms in learning and memory in Aplysia. Prog Brain Res 169:277-292.

Glanzman DL, Mackey SL, Hawkins RD, Dyke AM, Lloyd PE, Kandel ER (1989) Depletion of serotonin in the nervous system of Aplysia reduces the behavioral enhancement of gill withdrawal as well as the heterosynaptic facilitation produced by tail shock. J Neurosci 9:4200-4213.

Herbert JM, Augereau JM, Gleye J, Maffrand JP (1990) Chelerythrine is a potent and specific inhibitor of protein kinase C. Biochem Biophys Res Commun 172:993-999.

Hernandez AI, Blace N, Crary JF, Serrano PA, Leitges M, Libien JM, Weinstein G, Tcherapanov A, Sacktor TC (2003) Protein kinase M $\zeta$ synthesis from a brain mRNA encoding an independent protein kinase $\mathrm{C} \zeta$ catalytic domain. Implications for the molecular mechanism of memory. J Biol Chem 278:40305-40316.

Hiwasa T, Sawada T, Sakiyama S (1990) Cysteine proteinase inhibitors and rasgene products share the same biological activities including tranforming activity toward NIH3T3 mouse fibroblasts and the differentiationinducing activity toward PC12 rat pheochromocytoma cells. Carcinogenesis 11:75-80.

Houeland G, Nakhost A, Sossin WS, Castellucci VF (2007) PKC modulation of transmitter release by SNAP-25 at sensory-to-motor synapses in Aplysia. J Neurophysiol 97:134-143.

Hu JY, Meng X, Schacher S (2003) Redistribution of syntaxin mRNA in neuronal cell bodies regulates protein expression and transport during synapse formation and long-term synaptic plasticity. J Neurosci 23:1804-1815.

Hu JY, Glickman L, Wu F, Schacher S (2004) Serotonin regulates the secretion and autocrine action of a neuropeptide to activate MAPK required for long-term facilitation in Aplysia. Neuron 43:373-385.

Hu JY, Chen Y, Schacher S (2007) Protein kinase C regulates local synthesis and secretion of a neuropeptide required for activity-dependent longterm synaptic plasticity. J Neurosci 27:8927-8939.

Khoutorsky A, Spira ME (2005) Calcium-activated proteases are critical for refilling depleted vesicle stores in cultured sensory-motor synapses of Aplysia. Learn Mem 12:414-422.

Kruger KE, Sossin WS, Sacktor TC, Bergold PJ, Beushausen S, Schwartz JH (1991) Cloning and characterization of $\mathrm{Ca}^{2+}$-dependent and $\mathrm{Ca}^{2+}$-independent PKCs expressed in Aplysia sensory cells. J Neurosci 11:2303-2313.

Li Q, Roberts AC, Glanzman DL (2005) Synaptic facilitation and behavioral dishabituation in Aplysia: dependence upon release of $\mathrm{Ca}^{2+}$ from postsynaptic intracellular stores, postsynaptic exocytosis and modulation of postsynaptic AMPA receptor efficacy. J Neurosci 25:5623-5637.

Ling DS, Benardo LS, Serrano PA, Blace N, Kelly MT, Crary JF, Sacktor TC (2002) Protein kinase $M \zeta$ is necessary and sufficient for LTP maintenance. Nat Neurosci 5:295-296.

Ling DS, Benardo LS, Sacktor TC (2006) Protein kinase M $\zeta$ enhances excitatory synaptic transmission by increasing the number of active postsynaptic AMPA receptors. Hippocampus 16:443-452.
Lim T, Sossin WS (2006) Phosphorylation at the hydrophobic site of protein kinase C Apl II is increased during intermediate term facilitation. Neuroscience 141:277-285.

Lu X, Wyszynski M, Sheng M, Baudry M (2001) Proteolysis of glutamate receptor-interacting protein by calpain in rat brain: implications for synaptic plasticity. J Neurochem 77:1553-1560.

Mager DE, Kobrinsky E, Masoudieh A, Maltsev A, Abernethy DR, Soldatov NM (2007) Analysis of functional signaling domains from fluorescence imaging and the two-dimensional continuous wavelet transform. Biophys J 93:2900-2910.

Majewski H, Iannazzo L (1998) Protein kinase C: a physiological mediator of enhanced transmitter output. Prog Neurobiol 55:463-475.

Manseau F, Fan X, Hueftlein T, Sossin W, Castellucci VF (2001) $\mathrm{Ca}^{2+}$ independent protein kinase C Apl II mediates the serotonin-induced facilitation at depressed Aplysia sensorimotor synapses. J Neurosci 21:1247-1256.

Marinesco S, Carew TJ (2002) Serotonin release evoked by tail nerve stimulation in the CNS of Aplysia: characterization and relationship to heterosynaptic plasticity. J Neurosci 22:2299-2312.

Montarolo PG, Goelet P, Castellucci VF, Morgan J, Kandel ER, Schacher S (1986) A critical period for macromolecular synthesis in long-term heterosynaptic facilitation in Aplysia. Science 234:1249-1254.

Pastalkova E, Serrano P, Pinkhasova D, Wallace E, Fenton AA, Sacktor TC (2006) Storage of spatial information by the maintenance mechanism of LTP. Science 313:1141-1144.

Serrano P, Yao Y, Sacktor TC (2005) Persistent phosphorylation by protein kinase $\mathrm{M} \zeta$ maintains late-phase long-term potentiation. J Neurosci 25:1979-1984.

Shema R, Sacktor TC, Dudai Y (2007) Rapid erasure of long-term memory associations in the cortex by an inhibitor of PKM $\zeta$. Science 317:951-953.

Shobe JL, Zhao Y, Stough S, Ye X, Hsuan V, Martin KC, Carew TJ (2009) Temporal phases of activity-dependent plasticity and memory are mediated by compartmentalized routing of MAPK signaling in Aplysia sensory neurons. Neuron 61:113-125.

Sossin WS (1997) An autonomous kinase generated during long-term facilitation in Aplysia is related to the $\mathrm{Ca}^{2+}$-independent protein kinase C Apl II. Learn Mem 3:389-401.

Sossin WS (2007) Isoform specificity of protein kinase Cs in synaptic plasticity. Learn Mem 14:236-246.

Sossin WS, Diaz-Arrastia R, Schwartz JH (1993) Characterization of two isoforms of protein kinase $\mathrm{C}$ in the nervous system of Aplysia californica. J Biol Chem 268:5763-5768.

Sossin WS, Sacktor TC, Schwartz JH (1994) Persistent activation of protein kinase C during the development of long-term facilitation in Aplysia. Learn Mem 1:189-202.

Sossin WS, Fan X, Saberi F (1996) Expression and characterization of Aplysia protein kinase $\mathrm{C}$ : a negative regulatory role for the E region. J Neurosci 16:10-18.

Sutton MA, Carew TJ (2000) Parallel molecular pathways mediate expression of distinct forms of intermediate-term facilitation at tail sensorymotor synapses in Aplysia. Neuron 26:219-231.

Sutton MA, Bagnall MW, Sharma SK, Shobe J, Carew TJ (2004) Intermediateterm memory for site-specific sensitization in Aplysia is maintained by persistent activation of protein kinase C. J Neurosci 24:3600-3609.

Toullec D, Pianetti P, Coste H, Bellevergue P, Grand-Perret T, Ajakane M, Baudet V, Boissin P, Boursier E, Loriolle F, Dumahel L, Charon D, Kirilovsky J (1991) The bisindolylmaleimide GF 109203X is a potent and selective inhibitor of protein kinase C. J Biol Chem 266:15771-15781.

Villareal G, Li Q, Cai D, Glanzman DL (2007) The role of rapid, local postsynaptic protein synthesis in learning-related synaptic facilitation in Aplysia. Curr Biol 17:2073-2080.

Yao Y, Kelly MT, Sajikumar S, Serrano P, Tian D, Bergold PJ, Frey JU, Sacktor TC (2008) PKM $\zeta$ maintains late long-term potentiation by $\mathrm{N}$-ethylmaleimide-sensitive factor/GluR2-dependent trafficking of postsynaptic AMPA receptors. J Neurosci 28:7820-7827.

Zhao Y, Leal K, Abi-Farah C, Martin KC, Sossin WS, Klein M (2006) Isoform specificity of PKC translocation in living Aplysia sensory neurons and a role for $\mathrm{Ca}^{2+}$-dependent PKC APL I in the induction of intermediate-term facilitation. J Neurosci 26:8847-8856. 